\title{
USO DE MODELOS DIGITALES DE ELEVACIÓN Y DE SISTEMAS DE INFORMACIÓN GEOGRÁFICA EN LA MODELACIÓN HIDROLÓGICA
}

\author{
Ms. Graciela Pusineri \\ Profesora Cátedra SIG, Investigadora Dto. Hidrología ${ }_{(*)}$ \\ Ing. Raúl Pedraza \\ Profesor Cátedra Hidrología de Superficie, Investigador Dto. Hidrología \\ Director Dto. Hidrología ${ }_{(*)}$ \\ Ing. Cristóbal Lozeco \\ Profesor Cátedra Riego y Drenaje, Investigador Dto. Hidrología ${ }_{(*)}$ \\ ${ }_{\left({ }^{*}\right)}$ Facultad de Ingeniería y Ciencias Hídricas - Universidad Nacional del litoral
}

\section{RESUMEN}

La modelación hidrológica simula los procesos físicos que ocurren en un sistema hidrológico. La cuenca, vista como un sistema hidrológico, es la unidad espacial fundamental donde se desarrollan tales procesos. Los modelos hidrológicos utilizan ecuaciones que gobiernan el flujo de agua, las cuales relacionan variables de estado, de entrada/salida y parámetros.

Estos modelos necesitan información fisiográfica, tal como la configuración de la red de drenaje de la cuenca, delimitación y subdivisión de la misma en subcuencas, longitudes y pendientes de tramos de canal, superficies, longitudes y pendientes de subcuencas; así como información de tipo y cobertura del suelo. Estos parámetros eran obtenidos tradicionalmente de mapas analógicos o de mediciones de campo. Hace aproximadamente dos décadas, esta información ha comenzado a extraerse de Modelos Digitales de Elevación (MDE) y de Imágenes de Satélite.

Los Sistemas de Información Geográfica (SIG) juegan un papel fundamental en este sentido, no sólo en la integración de la información, sino en la capacidad que tienen para extraer automáticamente los parámetros necesarios para la modelación hidrológica, a través de operaciones espaciales usadas en algoritmos desarrollados para tal fin. Estos algoritmos permiten extraer información hidrológica del MDE, enlazar esta información a modelos o paquetes de modelos hidrológicos y realizar el postproceso, es decir recuperar las salidas de los modelos y graficarlas en el mismo ambiente SIG.

En este trabajo se describe la aplicación del SIG Arcview 3.2 y del programa AGWA (Automated Geospatial Watershed Assessment) a la cuenca del canal $1^{\circ}$ de Mayo en la ciudad de Esperanza (Prov. de Santa Fe). Como resultados del trabajo, se determinó el MDE de la cuenca y se extrajeron automáticamente los parámetros necesarios para la aplicación del modelo hidrológico Kineros2. La ventaja del procedimiento aplicado es que permite analizar en forma ágil distintas escalas espaciales para la desagregación de la cuenca.

\section{PALABRAS CLAVES}

Modelos Digitales de Elevación, Sistemas de Información Geográfica, Modelación Hidrológica. 


\section{INTRODUCCIÓN}

La simulación hidrológica comenzó en la década de los '50 con el advenimiento de las primeras computadoras digitales. Entre las variadas aplicaciones de los modelos de simulación hidrológica pueden mencionarse: el pronóstico de caudales, el diseño, el planeamiento (por ejemplo, para protección contra crecidas) y la extensión de registros de caudales.

Los primeros modelos fueron espacialmente concentrados, es decir, representaban la respuesta efectiva de una cuenca entera, sin caracterizar explícitamente la variabilidad espacial de la respuesta. Bajo este enfoque, la cuenca se discretiza en un conjunto de subcuencas, en donde los parámetros se consideran como homogéneos desde el punto de vista hidrológico. Los modelos espacialmente concentrados son todavía ampliamente usados, aunque no son capaces de representar la variabilidad espacial de los procesos hidrológicos y de los parámetros de la cuenca.

En las últimas dos décadas se está desarrollando una segunda generación de modelos de simulación hidrológica: los modelos distribuidos, los cuales representan explícitamente la variabilidad espacial de las características superficiales de la cuenca y de las precipitaciones. Se representan por mallas de celdas regulares de igual tamaño en donde la unidad de análisis es la celda. La resolución espacial está dada por el tamaño de la misma.

Ambos modelos tienen problemas para describir correctamente el comportamiento de la cuenca. Los concentrados porque hacen una simplificación demasiado grande y los distribuidos porque requieren enorme cantidad de datos que en la realidad no pueden ser obtenidos. Como resultado aparecen modelos que combinan aspectos de ambos, llamados cuasi distribuidos (Burns, 2004). Estos modelos dividen la cuenca en subcuencas (partiendo de las celdas), agrupándolas en unidades de análisis donde los parámetros tienen similares características hidrológicas.

Dos factores han favorecido la difusión de los modelos distribuidos y los cuasi distribuidos. Primero, la disponibilidad en soporte digital de los datos topográficos y de otros parámetros del suelo y cobertura. Segundo, el desarrollo de técnicas de rápido procesamiento de datos espaciales e imágenes de satélite.

Una de las estructuras de datos más importantes sobre la que se basan los procedimientos para el cálculo de los parámetros a ingresar en los modelos hidrológicos son los Modelos Digitales de Elevación (MDE). Los MDE son estructuras numéricas de datos que representan la distribución espacial de una variable cuantitativa y continua, la topografía (Felicísimo, 1994). Son estructuras muy ventajosas de donde puede extraerse gran parte de los parámetros necesarios para los análisis hidrológicos.

Los Sistemas de Información Geográfica (SIG) juegan un papel fundamental en este sentido, no sólo en la integración de la información, sino en la capacidad operatoria en procesamiento espacial que permite la extracción automática de los parámetros a incluir en la modelación hidrológica a partir del MDE. A su vez, los SIG permiten enlazar esta información a modelos o paquetes de modelos hidrológicos y realizar el postproceso, es decir recuperar las salidas de los modelos y graficarlas en el mismo ambiente SIG. Tal es el caso del programa AGWA (Automated Geospatial Watershed Assessment), módulo que se incorpora al SIG ArcView 3.x y permite usar este entorno para extraer parámetros de un MDE, armar archivos de entrada y correr un modelo hidrológico en segundo plano, para luego visualizar los resultados en el mismo entorno SIG. En la Fig. 1 se muestra una representación esquemática del vínculo entre la modelación hidrológica y los SIG 


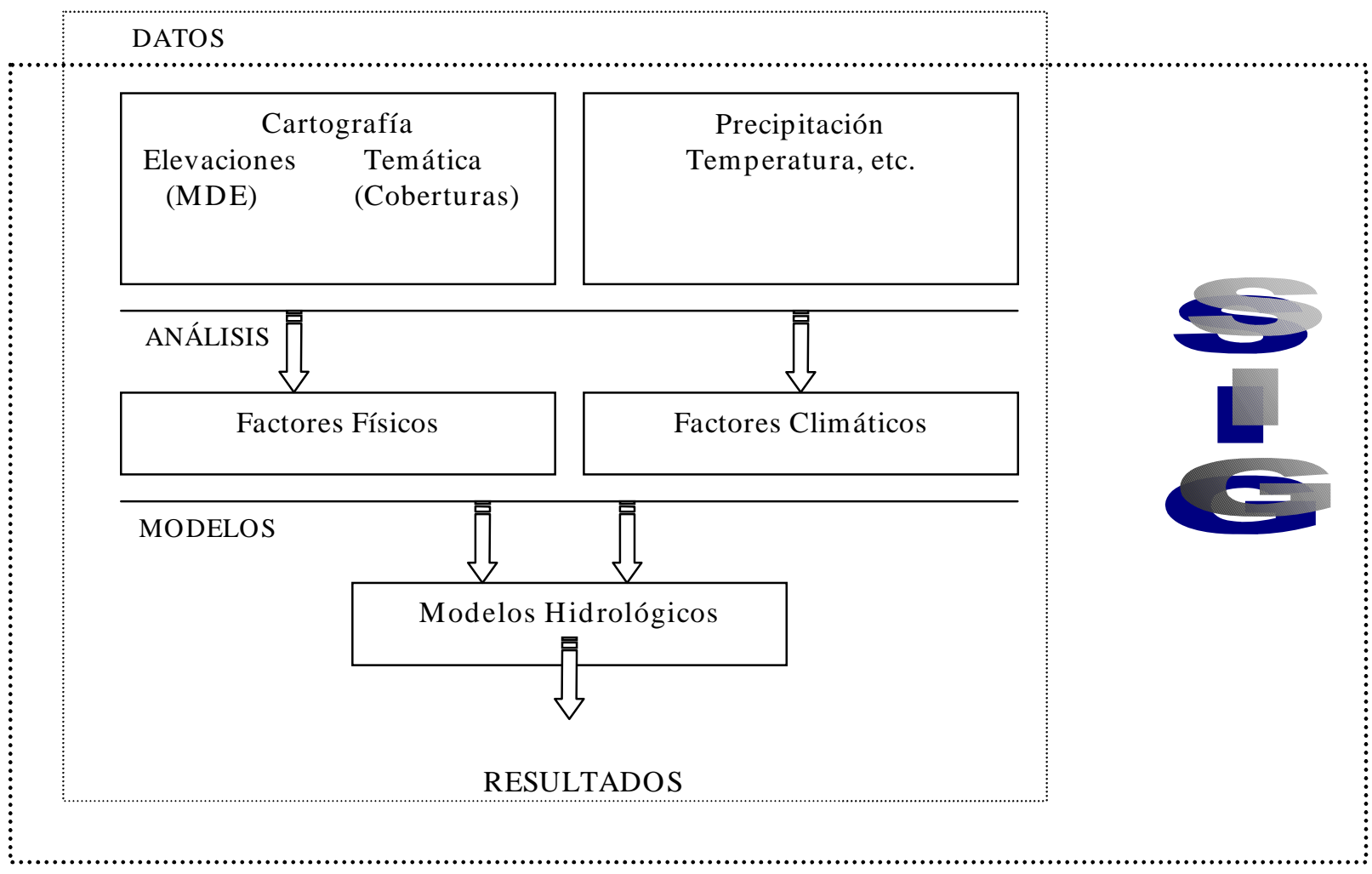

Fig. 1: Representación esquemática del vínculo entre la modelación hidrológica y los SIG (Olaya Ferrero, 2004), modificada por los autores

\section{OBJETIVO}

El objetivo de este trabajo es determinar en forma automática los parámetros requeridos por el modelo hidrológico Kineros 2, para distintas escalas espaciales, por medio de la aplicación del SIG Arcview 3.2 y el programa AGWA (Automated Geospatial Watershed Assessment) a la cuenca del canal $1^{\circ}$ de Mayo, ciudad de Esperanza (Prov. de Santa Fe).

\section{OBTENCIÓN AUTOMATIZADA DE PARÁMETROS HIDROLÓGICOS}

\subsection{Modelos Digitales del Terreno}

Los MDE son estructuras numéricas de datos que representan la distribución espacial de la altitud de la superficie del terreno (Felicísimo, 1994). Los más comunes son los de estructura tipo raster, que consisten en una malla o matriz de celdas cuadradas con la información de elevación en cada celda. Si bien existen otros, de tipo vectorial llamados TIN (Triangulated Irregular Network), no serán referidos en este trabajo.

Los SIG traen rutinas de cálculo para la generación de estas estructuras (raster o vectoriales). Estas rutinas traen diversos métodos de interpolación tales como: Kriging, Distancia Inversa, Mínima Curvatura, Regresión Polinomial, Funciones Radiales, Método Shepar, Interpolación Lineal por Triangulación y otros.

En los MDE tipo raster, la ubicación de la celda tiene implícito el concepto de fila, columna georreferenciada en algún sistema de coordenadas geográficas o de proyección plana conocida.

Un MDE tiene información suficiente para definir propiedades de la red de drenaje superficial y de la cuenca hidrológica. Para esto utilizan un conjunto de algoritmos basados en reglas de atracción repulsión en función de la distancia de una celda hacia sus vecinas más próximas. Estos algoritmos 
funcionan sobre una matriz de $\mathrm{n}$ dimensiones y esta organización matricial define un universo de celdas regulares que almacenan un valor. Este valor se actualiza en cada intervalo de tiempo, obedeciendo a reglas de propagación que dependen del valor de cada celda y de la de sus vecinos más próximos.

\subsection{Modificación del MDE original en zonas de llanuras}

Muchas veces es necesario modificar el MDE original, antes de comenzar con los procedimientos de cálculo de parámetros, para forzar el encauzamiento. Esta modificación es frecuentemente requerida en zonas llanas, en las cuales la preparación del MDE es mucho más complicada que en zonas de pendiente. Este procedimiento llamado, Stream - Burning (marcar a fuego los canales), consiste en imponer la red de drenaje al MDE, de manera que cuando se realice el análisis de flujo, se lo fuerce a seguir por allí hasta el punto de salida. La red de drenaje es un dato vectorial que se usa para verificar las direcciones de flujo calculadas, pero en este caso y previamente convertida a raster, se usará para modificar el MDE original, obteniendo de esta manera un conjunto de datos de mayor precisión.

\subsection{Procedimientos de Cálculo de Parámetros}

Diversos autores han desarrollado métodos para extraer información automática y delinear líneas de flujo y subcuencas a partir de los MDE. Muchas veces forman parte de módulos o subprogramas que funcionan en el entorno de los SIG y se encuentran disponibles en Internet. Básicamente consisten en:

1. Corrección de las zonas llanas y las depresiones que aparecen en el MDE origen (Fill Sink) Las zonas llanas son aquellas celdas o conjuntos de celdas del MDE origen que no tienen orientación. Hay varios algoritmos que corrigen esta situación, básicamente evaluando la tendencia de las celdas circundantes. Las depresiones son celdas a las que confluyen todas las circundantes y no encuentran salida. Para esta situación se efectúa un proceso de filtrado que suaviza el relieve, eliminado estas celdas atípicas a través de un proceso de rellenamiento (Fill Sink - rellenamiento de pozos).

2. Determinar la Dirección del Flujo hacia donde fluye cada celda.(Flowdirección) Consiste en determinar un patrón de movimiento a partir de las orientaciones de cada celda, calculadas en base a las pendientes. Se parte de una ventana de nxn celdas y se evalúa el comportamiento de la celda central con respecto a las circundantes. El principio básico de movimiento es el de menor potencial, es decir, hacia la dirección de máximas pendientes. Existen diversos algoritmos para este cálculo: el modelo D8, Rho8, FD8, Dinf, KRA y DEMON. El más comúnmente usado es el D8 que considera 8 direcciones de salida para una celda. La dirección del flujo es el camino de menor resistencia que recorrerá una gota de agua drenando por gravedad, desde un punto de mayor a uno de menor elevación.

3. Calcular la Acumulación del Flujo. (Flowacumulation)

Consiste en determinar todas las celdas que drenan a una en particular. Parte de evaluar el recorrido del flujo para cada celda a partir de la malla de direcciones de flujo. De esta manera aquellas celdas que tengan mayor acumulación del flujo, serán las que forman los canales de drenaje.

4. Identificar las Líneas de Flujo (Red de Drenaje)

Consiste en determinar las redes de drenaje a partir de la acumulación del flujo, considerando aquellas que tengan un determinado valor de acumulación (umbral) a partir del cual la celda comienza a drenar.

5. Delinear la cuenca a partir de la identificación de un punto de salida

Consiste en determinar el área aportante a un punto de salida o cierre de la cuenca, teniendo en cuenta la red de drenaje calculada en el punto anterior y el concepto de "umbral".

6. División de la cuenca en subcuencas,

Consiste en dividir a la cuenca aportante en subunidades hidrológicas. Existen varios criterios para realizar esta subdivisión, los que consideran puntos de cierre apoyándose en la red de 
drenaje y los que usan un área mínima llamada "Umbral". Esta última es la usada en el Programa Agwa y consiste en asignar un área mínima para las subcuencas como porcentaje del área total de la cuenca. Este proceso es en principio tentativo hasta dar con la resolución y detalle deseados.

7. Cálculo de propiedades de la cuenca y de las redes de drenaje

Se realiza una vectorización de los elementos hasta aquí determinados, como son cuenca, subcuencas y redes de drenaje y extracción del MDE de elevaciones de puntos máximos y mínimos para el cálculo de pendientes. Luego se calculan áreas y longitudes.

\section{PROGRAMA AGWA}

El programa AGWA (Automated Geospatial Watershed Assessment) es un conjunto de herramientas que trabajan en el entorno del SIG ArcView 3.x. Permiten la extracción automática de parámetros y la generación de los archivos de entrada a dos modelos: Kineros2 y Swat (modelos de simulación de escurrimiento en cuenca y perdidas por erosión respectivamente), la ejecución de estos modelos en segundo plano, y la recuperación de los resultados con la visualización en el ambiente SIG. La Fig.2 muestra la secuencia de pasos que se usan en Agwa para la simulación hidrológica.

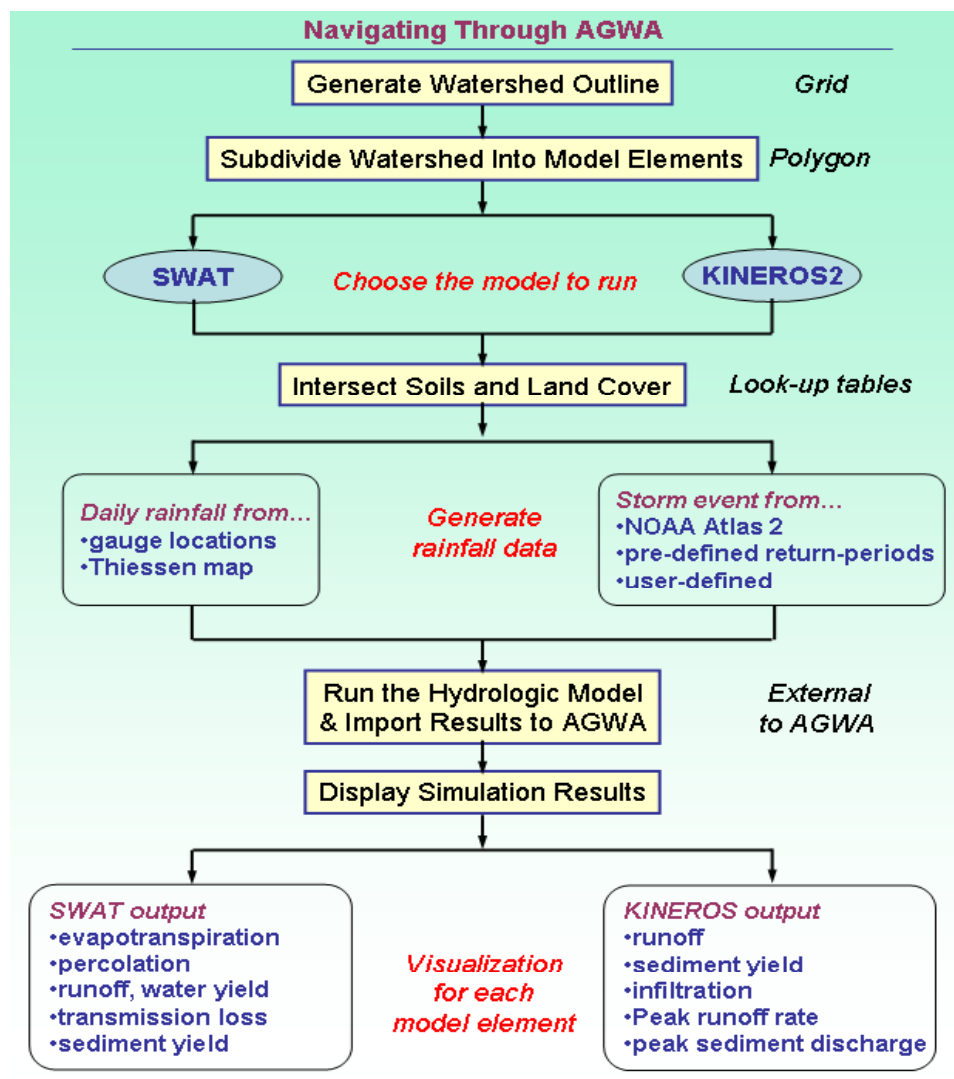

Fig. 2: Programa Agwa para Modelación Hidrológica, BURNS I.S. et al (2004)

El Programa Agwa requiere de la extensión Spatial Analyst para el manejo de estructuras de datos raster (mallas grilladas). Tiene herramientas para la extracción de parámetros fisiográficos a partir del MDE, de extracción de parámetros provenientes de mapas temáticos de tipo y cobertura de suelos, y para el tratamiento espacial de las precipitaciones.

En este trabajo se presenta una aplicación de la extracción de parámetros fisiográficos a partir del MDE, para la generación de los datos de entrada al modelo Kineros2 en la cuenca del canal $1^{\circ}$ de Mayo, en la ciudad de Esperanza (Provincia de Santa Fe). 


\section{AREA DE APLICACIÓN}

El área de estudio se ubica en el Departamento Las Colonias (Provincia de Santa Fe) y consiste en la cuenca de aportes al canal $1^{\circ}$ de Mayo, hasta su intersección con la calle Providencia, aproximadamente a $1000 \mathrm{~m}$ de la desembocadura en el río Salado. (Fig. 3). La cuenca tiene unas superficie de unas 1600 ha y comprende un sector urbanizado (ciudad de Esperanza y otro rural).

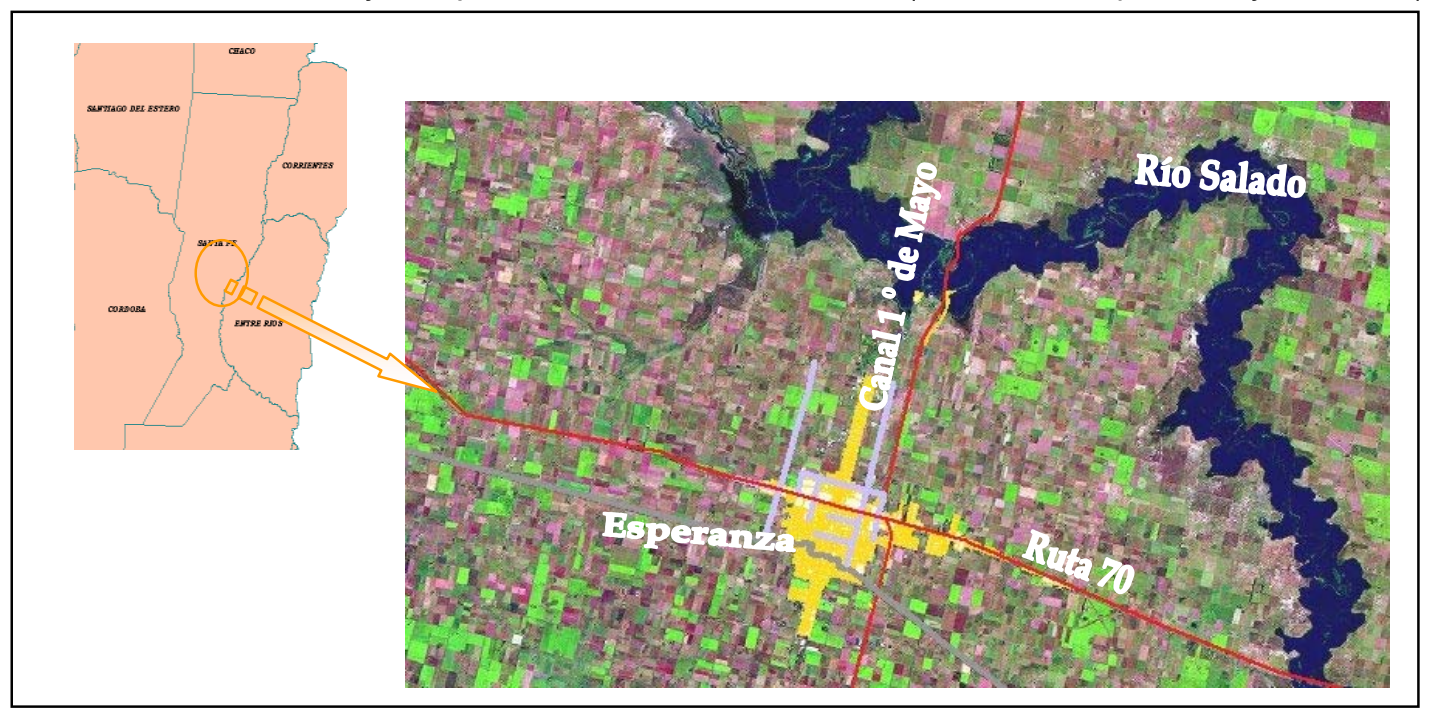

Fig. 3: Ubicación Geográfica del Canal 1 de Mayo. Dto. Las Colonias ,Santa Fe

Es una región de llanura suavemente ondulada, con vías de escurrimiento natural poco jerarquizadas y pendientes superficiales muy bajas, del orden del $0.1 \%$, orientadas hacia el río Salado.

En la zona rural, durante lluvias de gran magnitud, se satisface la capacidad de almacenamiento del suelo y se generan líneas de escurrimiento con dirección SW-NE, por vinculación de sucesivas depresiones hacia aguas abajo, hasta llegar al río Salado. La construcción de cunetas y canales de drenaje modifican sensiblemente la dinámica natural, debido a que las pendientes superficiales son muy suaves, generando transfluencias entre líneas de escurrimiento.

En la porción de área urbana que aporta al canal $1^{\circ}$ de Mayo, el agua de lluvia es conducida por un sistema combinado de conductos subterráneos y canales a cielo abierto.

\section{DESCRIPCIÓN DEL TRABAJO}

La construcción del MDE se realizó a partir de:

- curvas de nivel extraídas de la carta planialtimétrica Esperanza № 3160-26-1, escala 1:50000 del Instituto Geográfico Militar, IGM, 1959 y la carta planialtimétrica del área en estudio, en escala 1:20000 del Servicio de Catastro e Información Territorial de la Provincia de Santa Fe, SCIT, 1992.

- nivelación de detalle del casco urbano realizada en el estudio de diagnóstico de desagües pluviales de la ciudad de Esperanza $(\mathrm{FICH}, 1998)$.

- puntos acotados extraídos de planos a escalas mayores de trabajos específicos realizados por la Municipalidad de Esperanza.

A efectos de reproducir las modificaciones producidas por la acción antrópica y debido a la característica llana de la zona fue necesario modificar el MDE original. Esta modificación consistió en imponer una red de drenaje superficial, simplificada. Se representaron las trazas de los principales conductos subterráneos y canales a cielo abierto, de manera que en la simulación, el agua se vea forzada a seguir por estas líneas. Por otro lado, se impusieron dos "barreras" que representan a los terraplenes del FCGB y de la ruta 6 . Estos terraplenes actúan como divisoria de agua al $\mathrm{S}$ y $\mathrm{E}$ de la cuenca en estudio. (Fig 4). 
En este proceso se utilizaron herramientas de operatoria espacial del ArcView 3.x y el módulo Spatial Analyst. En primer lugar, se convirtió a formato de grillas la red de canales simplificada, la ruta y la vía férrea, obteniéndose los archivos grid_dren y grid_barreras. Las barreras se sumaron directamente al MDE original, pero para la imposición de la red de drenaje se efectuaron unos pasos previos. Se respetó la pendiente de fondo de los canales, para lo cual se realizó una interpolación de las cotas de fondo disponibles y luego se efectuaron cortes en el MDE, de modo de imprimir estas redes de drenaje con su pendiente de fondo original.

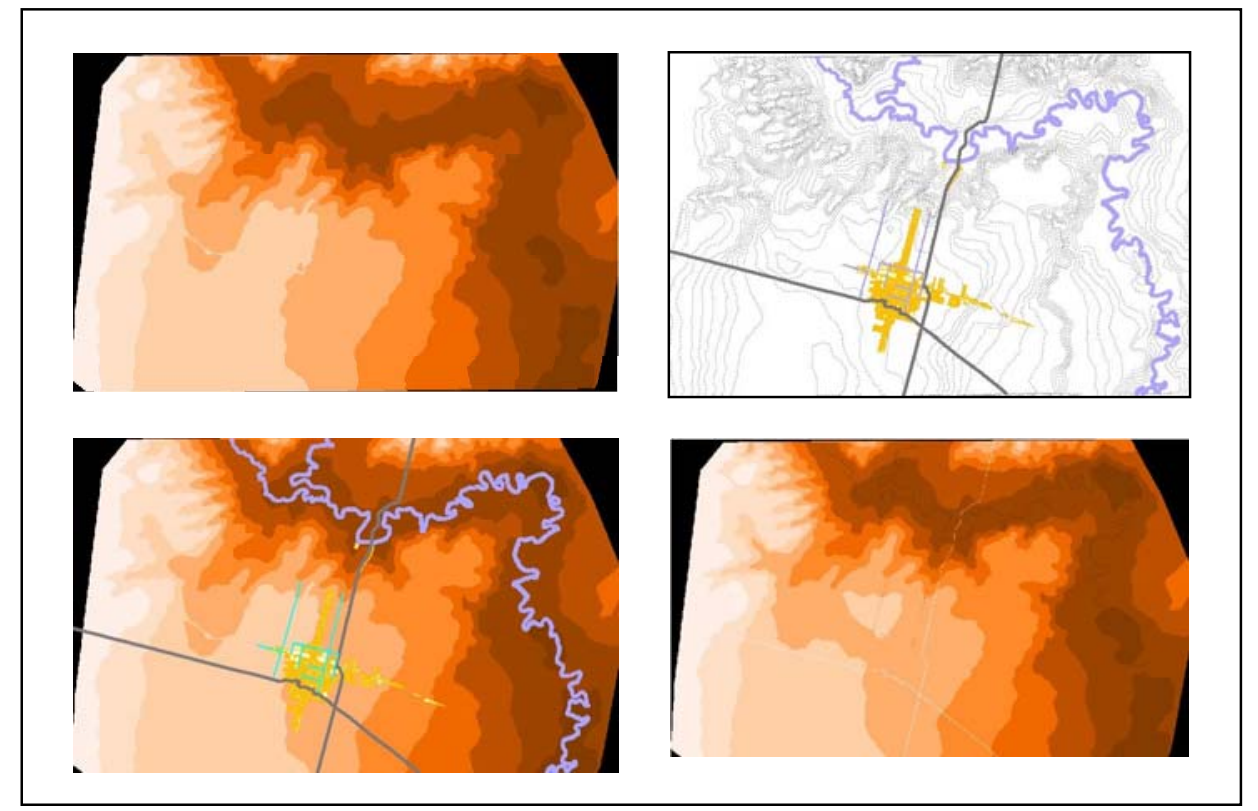

Fig. 4: Proceso de modificación del MDE original a través de la imposición de "barreras" y una red simplificad de canales

Con el MDE modificado se comenzó a trabajar con el módulo de Agwa dentro del entorno del SIG ArcView. Tal como se explica en el punto 3.3., se siguieron todos los pasos descriptos. En la Fig. 5 puede observarse la secuencia.

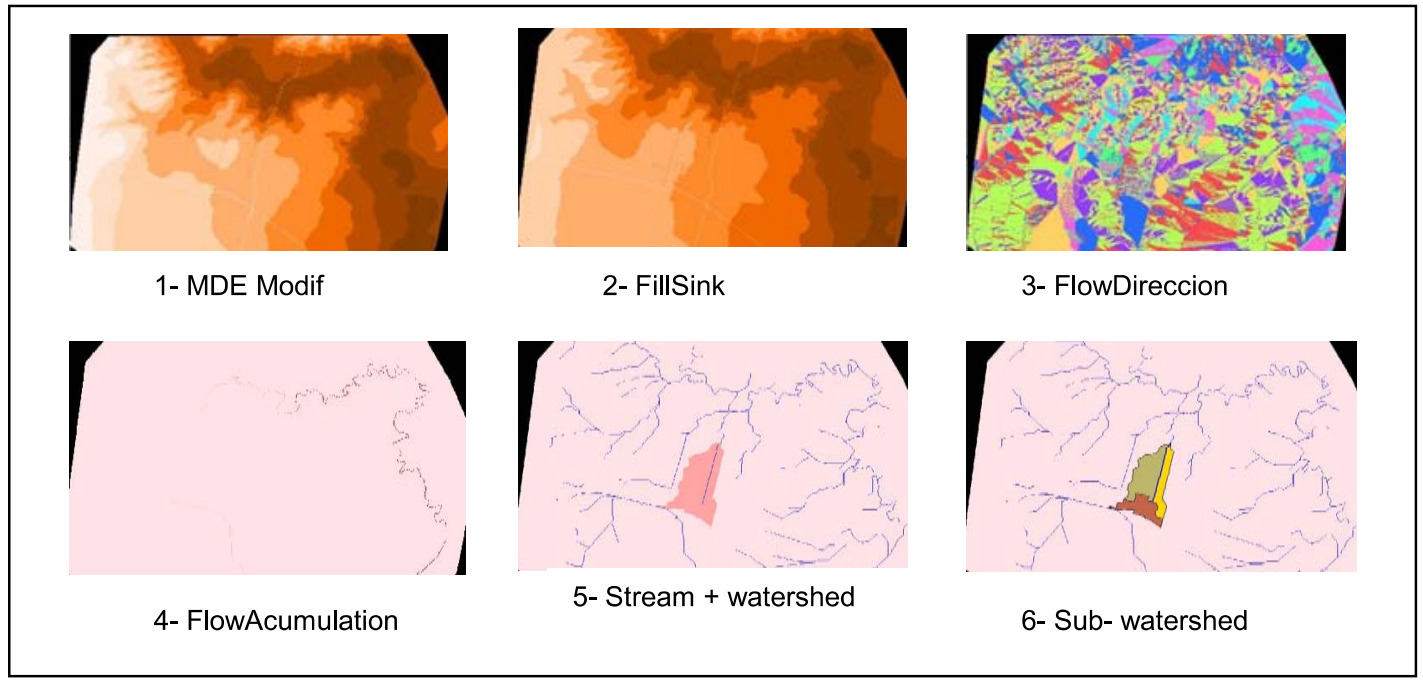

Fig . 5: Secuencia de Cálculo de parámetros fisiográficos por el programa AGWA, a partir del MDE modificado. 
Lo interesante de esta metodología, es que permite realizar distintos niveles de desagregaciones de la cuenca en subcuencas, una vez construido el MDE, lo que facilita el análisis de distintas escalas espaciales para la modelación. En este caso, se realizaron tres niveles de desagregación correspondiente a un área umbral del $20 \%, 10 \%$ y $7 \%$ respecto del área total de la cuenca. Puede observarse en la Fig. 6 que a medida que esta área disminuye, se genera una mayor cantidad de subcuencas y se representa la red de drenaje con mayor grado de detalle. Esto es así porque el área umbral marca el límite a partir del cual una celda comienza a drenar, cuanto más chica es el área umbral se está suponiendo que las celdas drenan con menor acumulación de flujo y por tanto son consideradas como parte de la red de drenaje.

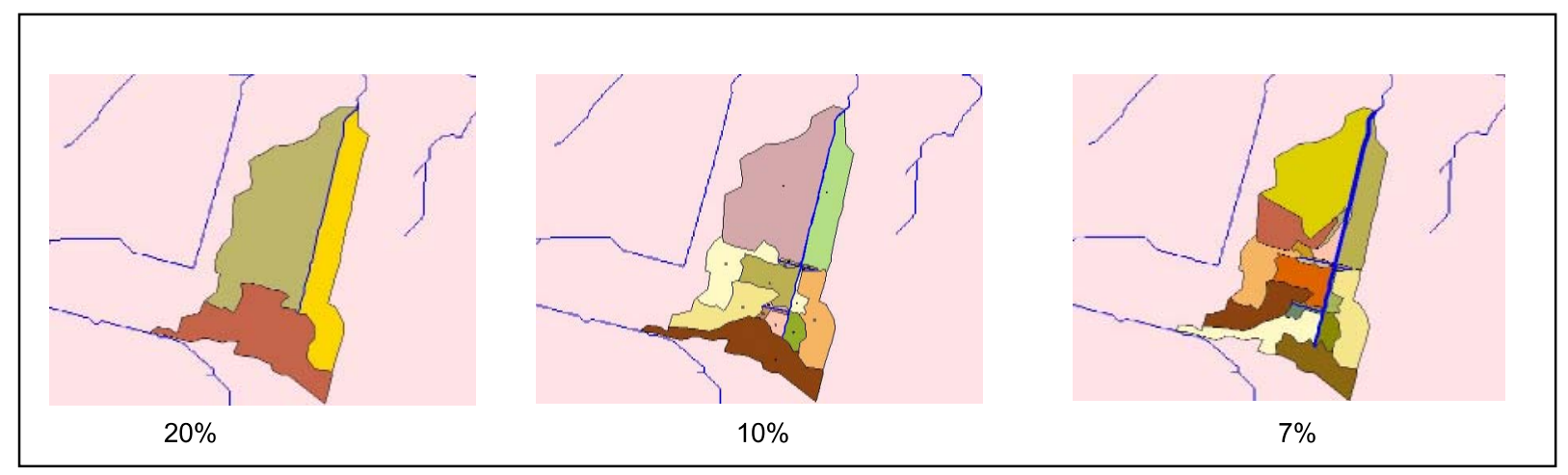

Fig. 6: Desagregación en subcuencas para un área del $20 \%, 10 \%$ y $7 \%$ respecto del área total de la cuenca

\section{CONCLUSIONES}

La determinación de un MDE en áreas de llanura urbanizadas requiere la modificación del MDE original, a efectos de contemplar tanto la red de drenaje superficial y subterránea; así como terraplenes viales y ferroviarios que actúan como divisorias de aguas.

En particular en este trabajo y por la potencialidad de los SIG en la operatoria espacial, se alteró el MDE original, imponiéndole la red de drenaje y las "barreras", procedimiento usado fundamentalmente para mejorar la representación de la dinámica hídrica, ya que al ser una zona llana, el MDE original no llega a representar bien las líneas de flujo para determinadas situaciones.

Se demuestra que el programa AGWA, desarrollado básicamente para cuencas de pendiente, tiene un buen desempeño en cuencas de llanura. Para ello, es necesario que el MDE original sea convenientemente modificado para representar la dinámica hídrica real de estas cuencas.

El avance en el tratamiento de la información permite un aumento en la resolución espacial de la modelación agilizando el análisis de distintas escalas espaciales para la desagregación de la cuenca en forma rápida y sencilla. Este análisis permite estudiar la variación espacial de factores que antes se consideraban como constantes.

\section{BIBLIOGRAFÍA}

AMERICAN SOCIETY OF CIVIL ENGINEERS (ASCE) Task Committee(1999) "GIS Modules and Distributed Models of the Watershed" ISBN: 0-7844-0443-7. Ed. ASCE. USA.

BURNS I.S. et al (2004)" Automated Geoespatial Watershed Assessment (AGWA) - A GIS based Hydrologic Modeling Tool - Documentation and User Manual. Versión 1.2.4.

http://www.tucson.ars.ag.gov/agwa/manual/AGWA/frame_controller.htmI Tucson, Arizona, USA. FELISÍCIMO A M (1994), Modelos Digitales de Terreno. Introducción y aplicación en las ciencias ambientales". http://etsimo.uniovi.es/feli/

MAIDMENT D. and DJOKIC D. (2000) " Hydrologic and Hydraulic Modeling support with Geographic Information System". ISBN:1-879102-80-3. Ed. ESRI. USA.

OLAYA FERRERO Víctor (2004) "Hidrología Computacional y Modelos Digitales del Terreno" . http://heart.sf.net./textos.html

PEDRAZA R, et al (1998) "Diagnóstico del Sistema de Drenaje Urbano de la ciudad de Esperanza, Santa Fe, Argentina". FICH - UNL. 